\title{
An Invitation to Split Quaternionic Analysis
}

\author{
Matvei Libine
}

May 23, 2018

\section{Introduction}

Six years after William Rowan Hamilton's discovery of quaternions, in 1849 James Cockle introduced the algebra of split quaternions [Co]. (He called them "coquaternions.") One way to define the split quaternions $\mathbb{H}_{\mathbb{R}}$ is by taking the standard generators for the algebra of quaternions $\mathbb{H}=\mathbb{R} 1 \oplus \mathbb{R} i \oplus \mathbb{R} j \oplus \mathbb{R} k$ and replacing the $i$ and $j$ with $\tilde{\iota}=\sqrt{-1} i$ and $\tilde{\jmath}=-\sqrt{-1} j$ respectively, so that $\mathbb{H}_{\mathbb{R}}=\mathbb{R} 1 \oplus \mathbb{R} \tilde{\iota} \oplus \mathbb{R} \tilde{\jmath} \oplus \mathbb{R} k$. Another way to realize split quaternions is as real $2 \times 2$ matrices. And yet another realization is

$$
\mathbb{H}_{\mathbb{R}}=\left\{\left(\begin{array}{ll}
z_{11} & z_{12} \\
z_{21} & z_{22}
\end{array}\right) ; z_{11}, z_{12}, z_{21}, z_{22} \in \mathbb{C}, z_{22}=\overline{z_{11}}, z_{21}=\overline{z_{12}}\right\}
$$

I. Frenkel initiated development of quaternionic analysis from the point of view of representation theory of the conformal group $S L(2, \mathbb{H})$ and its Lie algebra $\mathfrak{s l}(2, \mathbb{H})$. This approach has already been proven very fruitful and in our joint work [FL1] we push further the parallel with complex analysis and develop a rich theory. In particular we show that the quaternionic analogue of the Cauchy integral formula for the second order pole

$$
f^{\prime}(w)=\frac{1}{2 \pi i} \oint \frac{f(z) d z}{(z-w)^{2}}
$$

are the differential operator

$$
\mathrm{Mx} f=\nabla f \nabla-\square f^{+}
$$

defined on all holomorphic functions of four complex variables $f: \mathbb{H}_{\mathbb{C}} \rightarrow \mathbb{H}_{\mathbb{C}}$ and its integral presentation (note the square of the Fueter kernel)

$$
(\operatorname{Mx} f)(W)=\int_{C_{4}} \frac{(Z-W)^{-1}}{\operatorname{det}(Z-W)} \cdot f(Z) \cdot \frac{(Z-W)^{-1}}{\operatorname{det}(Z-W)} d Z^{4}
$$

where $d Z^{4}$ is the volume form, $C_{4}$ is a four cycle homologous to $U(2)=\left\{Z \in \mathbb{H}_{\mathbb{C}} ; Z^{*} Z=1\right\}$ sitting in the complexified quaternionic space $\mathbb{H}_{\mathbb{C}}=\mathbb{C} \otimes \mathbb{H}$. Since the constant functions on $\mathbb{C}$ are the holomorphic functions annihilated by the operator $d / d z: f(z) \mapsto f^{\prime}(z)$, their quaternionic analogue is the kernel of the operator $\mathrm{Mx}$, which turns out to be the space of solutions of a Euclidean version of the Maxwell equation for the gauge potential.

We also identify the Feynman integrals associated to the diagrams
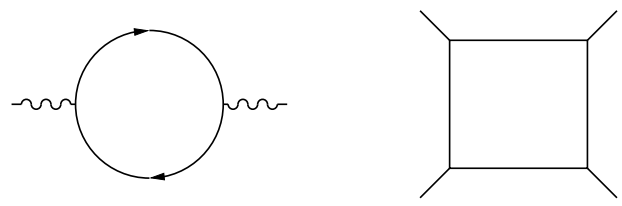

Feynman diagrams 
with the intertwining operators projecting certain natural unitary representations of $\mathfrak{s u}(2,2)$ onto their first irreducible components. Then we conjecture that the other Feynman integrals also admit an interpretation via quaternionic analysis and representation theory as the projectors onto the other irreducible components.

With this representation theoretic approach it quickly becomes evident that one has to consider the complexifications $\mathbb{H}_{\mathbb{C}}=\mathbb{C} \otimes \mathbb{H}$ and $\mathfrak{s l}(4, \mathbb{C})$ of $\mathbb{H}$ and $\mathfrak{s l}(2, \mathbb{H})$ and their real forms, such as the Minkowski space $\mathbb{M}$ and $\mathfrak{s u}(2,2)$ or the split quaternions $\mathbb{H}_{\mathbb{R}}$ and $\mathfrak{s l}(4, \mathbb{R})$. An important aspect of quaternionic analysis is its ability to compare representation theories of various real forms, and thus produce new results and make previously known results more explicit.

Just as (classical) quaternionic analysis is intimately related to the representation theory of $S U(2)$, split quaternionic analysis is related to the representation theory of $S L(2, \mathbb{R})$. The representation theory of $S L(2, \mathbb{R})$ is much richer than that of $S U(2)$ and exhibits most aspects of representations of higher rank real semisimple Lie groups. In particular, the group $S L(2, \mathbb{R})$ exhibits a subtle aspect of representation theory such as the separation of the discrete and continuous series of unitary representations:

$$
L^{2}(S L(2, \mathbb{R})) \simeq L_{\text {discr }}^{2}(S L(2, \mathbb{R})) \oplus L_{\text {cont }}^{2}(S L(2, \mathbb{R})) .
$$

We study this decomposition from the quaternionic point of view. The denominator of the Cauchy-Fueter kernel $\operatorname{det}\left(X-X_{0}\right)$ determines a region in $\mathbb{H}_{\mathbb{C}}=\mathbb{C} \otimes \mathbb{H}$

$$
\Omega=\left\{X_{0} \in \mathbb{H}_{\mathbb{C}} ; \operatorname{det}\left(X-X_{0}\right) \neq 0 \quad \forall X \in \mathbb{H}_{\mathbb{R}} \text { with } \operatorname{det}(X)=1\right\} .
$$

Loosely speaking, this region can be broken into several connected components, two of which are open Ol'shanskii semigroups of $G L(2, \mathbb{C})$. Then the Cauchy-Fueter kernel $\frac{\left(X-X_{0}\right)^{-1}}{\operatorname{det}\left(X-X_{0}\right)}$ on $\mathbb{H}_{\mathbb{R}}$ can be expanded in terms of the $K$-types of the discrete or continuous series of $S L(2, \mathbb{R})$ depending on the choice of connected component of $\Omega$ containing $X_{0}$. It follows that the projectors onto the discrete and continuous series of $S L(2, \mathbb{R})$ can be expressed as

$$
\begin{aligned}
& \left(\mathrm{P}^{\text {discr }}\right) f\left(X_{0}\right)=\frac{1}{2 \pi^{2}} \int_{C_{\text {discr }}} \frac{\left(X-X_{0}\right)^{-1}}{\operatorname{det}\left(X-X_{0}\right)} \cdot D z \cdot f(X), \\
& \left(\mathrm{P}^{\text {cont }}\right) f\left(X_{0}\right)=\frac{1}{2 \pi^{2}} \int_{C_{\text {cont }}} \frac{\left(X-X_{0}\right)^{-1}}{\operatorname{det}\left(X-X_{0}\right)} \cdot D z \cdot f(X),
\end{aligned}
$$

where $C_{d i s c r}$ and $C_{\text {cont }}$ are certain three cycles in $\Omega$. Note that these two integrals are identical to the Cauchy-Fueter formula, except for the choice of contours of integration $C_{d i s c r}$ and $C_{\text {cont }}$. Moreover, by choosing appropriate cycles we can even get projectors onto the holomorphic discrete series and antiholomorphic discrete series. Such a geometric description of the decomposition (11) fits well into the Gelfand-Gindikin program initiated in GG]. This relationship between quaternionic analysis and the separation of the series for $S L(2, \mathbb{R})$ will be the subject of our upcoming paper [FL2].

In view of how many connections to mathematical physics (classical) quaternionic analysis has (see, for example, GT]), it is almost guaranteed that split quaternionic analysis will have them as well. At this point it is worth mentioning two very recent physics papers [ACCK] and [MS] stressing the importance of the $(2,2)$ signature of the split quaternions $\mathbb{H}_{\mathbb{R}}$ as opposed to the traditional Lorentzian signature of the Minkowski space $\mathbb{M}$.

Once split quaternionic analysis is sufficiently developed, it can be generalized in many different ways. Perhaps the most obvious direction is to extend the new results to higher 
dimensions. There is a generalization of quaternionic analysis known as Clifford analysis. Let $\mathfrak{C r}(V)$ be a Clifford algebra over a real finite-dimensional vector space $V$ with a nondegenerate quadratic form $Q(x)$. Then on $\mathbb{R} \oplus V$ we can introduce Dirac operators $D$ and $D^{+}$with coefficients in $\mathfrak{C l}(V)$ so that $D D^{+}=D^{+} D$ is the wave operator on $\mathbb{R} \oplus V$ with symbol $x^{2}-Q$. We define a differentiable function $f: \mathbb{R} \oplus V \rightarrow \mathfrak{C l}(V)$ to be (left) Clifford analytic (or monogenic) if $D^{+} f=0$. (We think of it as an analogue of the Cauchy-Riemann equations.) Slightly more generally, we can define Clifford analytic functions with values in a $\mathfrak{C r}(V)$-module. When the quadratic form $Q$ is negative definite there is a well developed theory of Clifford analytic functions called Clifford analysis (see, for example, [BDS], [DSS] and [GM]). This theory generalizes (classical) quaternionic analysis. Note that in this case $D D^{+}$is the Laplacian on $\mathbb{R} \oplus V$, hence the components of Clifford analytic functions are harmonic. Clifford analysis has deep connections with harmonic analysis, representations of spin groups and index theory of Dirac operators.

Methods developed in split quaternionic analysis will extend to analysis of Clifford analytic functions associated with quadratic forms of arbitrary signature. Thus we can consider split Clifford analysis as a "real form" of complexified Clifford analysis, which in turn was introduced by Ryan in $[R]$. On the other hand, the use of the wave equation for the study of harmonic analysis on a hyperboloid goes back to Strichartz [St]. More recently, Kobayashi and Ørsted KoØ] study representations of $O(p+1, q+1)$ in the space of solutions of the wave equation $\square_{p, q} \varphi=0$ on $\mathbb{R}^{p+q}$. In this light it is natural to expect that this split Clifford analysis will yield new results relating solutions of the wave equation, representation theory and index theory of Dirac operators. In particular, we expect to obtain concrete realizations of representations of $O(p+1, q+1)$ in the space of solutions of Clifford analytic functions. Since Clifford analysis is widely used by mathematical physicists, it is very likely that they will find the split version at least as useful as the classical one.

In this article we approach the split quaternions $\mathbb{H}_{\mathbb{R}}$ as a real form of $\mathbb{H}_{\mathbb{C}}$, introduce the notion of regular functions and give two different analogues of the Cauchy-Fueter formula valid for different classes of functions. This is done in parallel with (classical) quaternionic analysis. We conclude the paper with an outline of our derivation of the projectors $\mathrm{P}^{\text {discr }}$ and $\mathrm{P}^{\text {cont }}$ onto the discrete and continuous series of $S L(2, \mathbb{R})$. Some contemporary reviews of quaternionic analysis are given in $[\mathrm{Su}]$ and [CSSS].

\section{The Quaternionic Spaces $\mathbb{H}_{\mathbb{C}}, \mathbb{H}_{\mathbb{R}}$ and $\mathbb{M}$}

In this article we use notations established in [FL1]. In particular, $e_{0}, e_{1}, e_{2}, e_{3}$ denote the units of the classical quaternions $\mathbb{H}$ corresponding to the more familiar $1, i, j, k$ (we reserve the symbol $i$ for $\sqrt{-1} \in \mathbb{C}$ ). Thus $\mathbb{H}$ is an algebra over $\mathbb{R}$ generated by $e_{0}, e_{1}, e_{2}, e_{3}$, and the multiplicative structure is determined by the rules

$$
e_{0} e_{i}=e_{i} e_{0}=e_{i}, \quad\left(e_{i}\right)^{2}=-e_{0}, \quad e_{i} e_{j}=-e_{i} e_{j}, \quad 1 \leq i<j \leq 3,
$$

and the fact that $\mathbb{H}$ is a division ring. Next we consider the algebra of complexified quaternions $\mathbb{H}_{\mathbb{C}}=\mathbb{C} \otimes \mathbb{H}$ (also known as biquaternions). We define a complex conjugation on $\mathbb{H}_{\mathbb{C}}$ with respect to $\mathbb{H}$ :

$$
Z=z^{0} e_{0}+z^{1} e_{1}+z^{2} e_{2}+z^{3} e_{3} \quad \mapsto \quad Z^{c}=\overline{z^{0}} e_{0}+\overline{z^{1}} e_{1}+\overline{z^{2}} e_{2}+\overline{z^{3}} e_{3}, \quad z^{0}, z^{1}, z^{2}, z^{3} \in \mathbb{C},
$$

so that $\mathbb{H}=\left\{Z \in \mathbb{H}_{\mathbb{C}} ; Z^{c}=Z\right\}$. The quaternionic conjugation on $\mathbb{H}_{\mathbb{C}}$ is defined by:

$$
Z=z^{0} e_{0}+z^{1} e_{1}+z^{2} e_{2}+z^{3} e_{3} \quad \mapsto \quad Z^{+}=z^{0} e_{0}-z^{1} e_{1}-z^{2} e_{2}-z^{3} e_{3}, \quad z^{0}, z^{1}, z^{2}, z^{3} \in \mathbb{C} ;
$$


it is an anti-involution:

$$
(Z W)^{+}=W^{+} Z^{+}, \quad \forall Z, W \in \mathbb{H}_{\mathbb{C}}
$$

We will also use an involution

$$
\left.Z \mapsto Z^{-}=-e_{3} Z e_{3} \quad \text { (conjugation by } e_{3}\right) .
$$

Then the complex conjugation, the quaternionic conjugation and the involution $Z \mapsto Z^{-}$commute with each other.

In this article we will be primarily interested in the space of split quaternions $\mathbb{H}_{\mathbb{R}}$ which is a real form of $\mathbb{H}_{\mathbb{C}}$ defined by

$$
\mathbb{H}_{\mathbb{R}}=\left\{Z \in \mathbb{H}_{\mathbb{C}} ; Z^{c-}=Z\right\}=\left\{\mathbb{R} \text {-span of } e_{0}, \tilde{e}_{1}=i e_{1}, \tilde{e}_{2}=-i e_{2}, e_{3}\right\}
$$

We will also consider the Minkowski space $\mathbb{M}$ which we regard as another real form of $\mathbb{H}_{\mathbb{C}}$ :

$$
\mathbb{M}=\left\{Z \in \mathbb{H}_{\mathbb{C}} ; Z^{c+}=-Z\right\}
$$

$\mathbb{M}$ is spanned over $\mathbb{R}$ by $\tilde{e}_{0}=-i e_{0}, e_{1}, e_{2}, e_{3}$.

On $\mathbb{H}_{\mathbb{C}}$ we have a quadratic form $N$ defined by

$$
N(Z)=Z Z^{+}=Z^{+} Z=\left(z^{0}\right)^{2}+\left(z^{1}\right)^{2}+\left(z^{2}\right)^{2}+\left(z^{3}\right)^{2}
$$

hence

$$
Z^{-1}=\frac{Z^{+}}{N(Z)}
$$

The corresponding symmetric bilinear form on $\mathbb{H}_{\mathbb{C}}$ is

$$
\langle Z, W\rangle=\frac{1}{2} \operatorname{Tr}\left(Z^{+} W\right)=\frac{1}{2} \operatorname{Tr}\left(Z W^{+}\right), \quad Z, W \in \mathbb{H}_{\mathbb{C}}
$$

where $\operatorname{Tr} Z=2 z^{0}=Z+Z^{+}$. When this quadratic form is restricted to $\mathbb{H}, \mathbb{H}_{\mathbb{R}}$ and $\mathbb{M}$, it has signature $(4,0),(2,2)$ and $(3,1)$ respectively. The real forms $\mathbb{H}$ and $\mathbb{M}$ have been studied in FL1, and the signature $(1,3)$ is equivalent to $(3,1)$. In this article we study the real form $\mathbb{H}_{\mathbb{R}}$ realizing the only remaining signature $(2,2)$.

We will use the standard matrix realization of $\mathbb{H}$ so that

$$
e_{0}=\left(\begin{array}{cc}
1 & 0 \\
0 & 1
\end{array}\right), \quad e_{1}=\left(\begin{array}{cc}
0 & -i \\
-i & 0
\end{array}\right), \quad e_{2}=\left(\begin{array}{cc}
0 & -1 \\
1 & 0
\end{array}\right), \quad e_{3}=\left(\begin{array}{cc}
-i & 0 \\
0 & i
\end{array}\right)
$$

and

$$
\mathbb{H}=\left\{Z \in \mathbb{H}_{\mathbb{C}} ; Z^{c}=Z\right\}=\left\{Z=\left(\begin{array}{ll}
z_{11} & z_{12} \\
z_{21} & z_{22}
\end{array}\right) \in \mathbb{H}_{\mathbb{C}} ; z_{22}=\overline{z_{11}}, z_{21}=-\overline{z_{12}}\right\} .
$$

Then $\mathbb{H}_{\mathbb{C}}$ can be identified with the algebra of all complex $2 \times 2$ matrices:

$$
\mathbb{H}_{\mathbb{C}}=\left\{Z=\left(\begin{array}{ll}
z_{11} & z_{12} \\
z_{21} & z_{22}
\end{array}\right) ; z_{i j} \in \mathbb{C}\right\}
$$

the quadratic form $N(Z)$ becomes $\operatorname{det} Z$ and the involution $Z \mapsto Z^{-}$becomes

$$
Z=\left(\begin{array}{cc}
z_{11} & z_{12} \\
z_{21} & z_{22}
\end{array}\right) \mapsto Z^{-}=\left(\begin{array}{cc}
1 & 0 \\
0 & -1
\end{array}\right) Z\left(\begin{array}{cc}
1 & 0 \\
0 & -1
\end{array}\right)=\left(\begin{array}{cc}
z_{11} & -z_{12} \\
-z_{21} & z_{22}
\end{array}\right)
$$


The split quaternions $\mathbb{H}_{\mathbb{R}}$ and the Minkowski space $\mathbb{M}$ have matrix realizations

$$
\mathbb{H}_{\mathbb{R}}=\left\{Z=\left(\begin{array}{ll}
z_{11} & z_{12} \\
z_{21} & z_{22}
\end{array}\right) \in \mathbb{H}_{\mathbb{C}} ; z_{22}=\overline{z_{11}}, z_{21}=\overline{z_{12}}\right\}
$$

and

$$
\mathbb{M}=\left\{Z=\left(\begin{array}{ll}
z_{11} & z_{12} \\
z_{21} & z_{22}
\end{array}\right) \in \mathbb{H}_{\mathbb{C}} ; z_{11}, z_{22} \in i \mathbb{R}, z_{21}=-\overline{z_{12}}\right\}
$$

From this realization it is easy to see that the split quaternions form an algebra over $\mathbb{R}$ isomorphic to $\mathfrak{g l}(2, \mathbb{R})$, the invertible elements in $\mathbb{H}_{\mathbb{R}}$, denoted by $\mathbb{H}_{\mathbb{R}}^{\times}$, are nothing else but $G L(2, \mathbb{R})$. We regard $S L(2, \mathbb{C})$ as a quadric $\{N(Z)=1\}$ in $\mathbb{H}_{\mathbb{C}}$, and we also regard $S U(1,1) \simeq S L(2, \mathbb{R})$ as the set of real points of this quadric:

$$
\begin{aligned}
& S U(1,1)=\left\{Z \in \mathbb{H}_{\mathbb{R}} ; N(Z)=1\right\} \\
& =\left\{Z=\left(\begin{array}{ll}
z_{11} & z_{12} \\
\overline{z_{12}} & \overline{z_{11}}
\end{array}\right) \in \mathbb{H}_{\mathbb{R}} ; \operatorname{det} Z=\left|z_{11}\right|^{2}-\left|z_{12}\right|^{2}=1\right\} .
\end{aligned}
$$

The algebra of split quaternions $\mathbb{H}_{\mathbb{R}}$ is spanned over $\mathbb{R}$ by the four matrices

$$
e_{0}=\left(\begin{array}{cc}
1 & 0 \\
0 & 1
\end{array}\right), \quad \tilde{e}_{1}=\left(\begin{array}{cc}
0 & 1 \\
1 & 0
\end{array}\right), \quad \tilde{e}_{2}=\left(\begin{array}{cc}
0 & i \\
-i & 0
\end{array}\right), \quad e_{3}=\left(\begin{array}{cc}
-i & 0 \\
0 & i
\end{array}\right)
$$

so

$$
\mathbb{H}_{\mathbb{R}}=\left\{x^{0} e_{0}+x^{1} \tilde{e}_{1}+x^{2} \tilde{e}_{2}+x^{3} e_{3}=\left(\begin{array}{ll}
x^{0}-i x^{3} & x^{1}+i x^{2} \\
x^{1}-i x^{2} & x^{0}+i x^{3}
\end{array}\right) ; x^{0}, x^{1}, x^{2}, x^{3} \in \mathbb{R}\right\} .
$$

The quaternionic conjugation in this basis becomes

$$
e_{0}^{+}=e_{0}, \quad \tilde{e}_{1}^{+}=-\tilde{e}_{1}, \quad \tilde{e}_{2}^{+}=-\tilde{e}_{2}, \quad e_{3}^{+}=-e_{3} .
$$

The multiplication rules for $\mathbb{H}_{\mathbb{R}}$ are:

$$
\begin{gathered}
e_{0} \text { commutes with all elements of } \mathbb{H}_{\mathbb{R}} \\
\tilde{e}_{1}, \tilde{e}_{2}, e_{3} \text { anti-commute, } \\
e_{0}^{2}=\tilde{e}_{1}^{2}=\tilde{e}_{2}^{2}=e_{0}, \quad e_{3}^{2}=-e_{0}, \\
\tilde{e}_{1} \tilde{e}_{2}=e_{3}, \quad \tilde{e}_{2} e_{3}=-\tilde{e}_{1}, \quad e_{3} \tilde{e}_{1}=-\tilde{e}_{2} .
\end{gathered}
$$

The elements $e_{0}, \tilde{e}_{1}, \tilde{e}_{2}, e_{3}$ are orthogonal with respect to the bilinear form (2) and $\left\langle e_{0}, e_{0}\right\rangle=$ $\left\langle e_{3}, e_{3}\right\rangle=1,\left\langle\tilde{e}_{1}, \tilde{e}_{1}\right\rangle=\left\langle\tilde{e}_{2}, \tilde{e}_{2}\right\rangle=-1$.

The (classical) quaternions $\mathbb{H}$ are oriented so that $\left\{e_{0}, e_{1}, e_{2}, e_{3}\right\}$ is a positive basis. Let $d V=d z^{0} \wedge d z^{1} \wedge d z^{2} \wedge d z^{3}$ be the holomorphic 4-form on $\mathbb{H}_{\mathbb{C}}$ determined by $d V\left(e_{0}, e_{1}, e_{2}, e_{3}\right)=1$, then the restriction $\left.d V\right|_{\mathbb{H}}$ is the Euclidean volume form corresponding to $\left\{e_{0}, e_{1}, e_{2}, e_{3}\right\}$. On the other hand, the restriction $\left.d V\right|_{\mathbb{H}_{\mathbb{R}}}$ is also real-valued and hence determines an orientation on $\mathbb{H}_{\mathbb{R}}$ so that $\left\{e_{0}, \tilde{e}_{1}, \tilde{e}_{2}, e_{3}\right\}$ becomes a positively oriented basis. Define a norm on $\mathbb{H}_{\mathbb{C}}$ by

$$
\|Z\|=\frac{1}{\sqrt{2}} \sqrt{\left|z_{11}\right|^{2}+\left|z_{12}\right|^{2}+\left|z_{21}\right|^{2}+\left|z_{22}\right|^{2}}, \quad Z=\left(\begin{array}{cc}
z_{11} & z_{12} \\
z_{21} & z_{22}
\end{array}\right) \in \mathbb{H}_{\mathbb{C}}
$$

so that $\left\|e_{i}\right\|=1,0 \leq i \leq 3$.

In [FL1] we defined a holomorphic 3 -form on $\mathbb{H}_{\mathbb{C}}$ with values in $\mathbb{H}_{\mathbb{C}}$

$$
D z=e_{0} d z^{1} \wedge d z^{2} \wedge d z^{3}-e_{1} d z^{0} \wedge d z^{2} \wedge d z^{3}+e_{2} d z^{0} \wedge d z^{1} \wedge d z^{3}-e_{3} d z^{0} \wedge d z^{1} \wedge d z^{2}
$$


characterized by the property

$$
\begin{aligned}
&\left\langle Z_{1}, D z\left(Z_{2}, Z_{3}, Z_{4}\right)\right\rangle=\frac{1}{2} \operatorname{Tr}\left(Z_{1}^{+}, D z\left(Z_{2}, Z_{3}, Z_{4}\right)\right)=d V\left(Z_{1}, Z_{2}, Z_{3}, Z_{4}\right) \\
& \forall Z_{1}, Z_{2}, Z_{3}, Z_{4} \in \mathbb{H}_{\mathbb{C}} .
\end{aligned}
$$

Let $D x=\left.D z\right|_{\mathbb{H}_{\mathbb{R}}}$ and $D \tilde{x}=\left.D z\right|_{\mathbb{H}}$.

Proposition 1 The 3-form Dx takes values in $\mathbb{H}_{\mathbb{R}}$. If we write $X=x^{0} e_{0}+x^{1} \tilde{e}_{1}+x^{2} \tilde{e}_{2}+x^{3} e_{3} \in$ $\mathbb{H}_{\mathbb{R}}, x^{0}, x^{1}, x^{2}, x^{3} \in \mathbb{R}$, then $D x$ is given explicitly by

$$
D x=e_{0} d x^{1} \wedge d x^{2} \wedge d x^{3}+\tilde{e}_{1} d x^{0} \wedge d x^{2} \wedge d x^{3}-\tilde{e}_{2} d x^{0} \wedge d x^{1} \wedge d x^{3}-e_{3} d x^{0} \wedge d x^{1} \wedge d x^{2} .
$$

Remark 2 Clearly, the form Dx satisfies the property

$$
\begin{array}{r}
\left\langle X_{1}, D x\left(X_{2}, X_{3}, X_{4}\right)\right\rangle=\frac{1}{2} \operatorname{Tr}\left(X_{1}^{+}, D x\left(X_{2}, X_{3}, X_{4}\right)\right)=d V\left(X_{1}, X_{2}, X_{3}, X_{4}\right), \\
\forall X_{1}, X_{2}, X_{3}, X_{4} \in \mathbb{H}_{\mathbb{R}},
\end{array}
$$

which could be used to define it.

It is also worth mentioning that in terms of the coordinates $Z=\left(\begin{array}{ll}z_{11} & z_{12} \\ z_{21} & z_{22}\end{array}\right)$ on $\mathbb{H}_{\mathbb{C}}, z_{i j} \in \mathbb{C}$,

$$
D z=\frac{1}{2}\left(\begin{array}{cc}
-d z_{11} \wedge d z_{12} \wedge d z_{21} & -d z_{11} \wedge d z_{12} \wedge d z_{22} \\
d z_{11} \wedge d z_{21} \wedge d z_{22} & d z_{12} \wedge d z_{21} \wedge d z_{22}
\end{array}\right)
$$

where we write $z_{i j}=x_{i j}+i y_{i j}, x_{i j}, y_{i j} \in \mathbb{R}$, and $d z_{i j}=d x_{i j}+i d y_{i j}$.

Let $U \subset \mathbb{H}_{\mathbb{R}}$ be an open region with piecewise smooth boundary $\partial U$. We give a canonical orientation to $\partial U$ as follows. The positive orientation of $U$ is determined by $\left\{e_{0}, \tilde{e}_{1}, \tilde{e}_{2}, e_{3}\right\}$. Pick a smooth point $p \in \partial U$ and let $\overrightarrow{n_{p}}$ be a non-zero vector in $T_{p} \mathbb{H}_{\mathbb{R}}$ perpendicular to $T_{p} \partial U$ and pointing outside of $U$. Then $\left\{\overrightarrow{\tau_{1}}, \overrightarrow{\tau_{2}}, \overrightarrow{\tau_{3}}\right\} \subset T_{p} \partial U$ is positively oriented in $\partial U$ if and only if $\left\{\overrightarrow{n_{p}}, \overrightarrow{\tau_{1}}, \overrightarrow{\tau_{2}}, \overrightarrow{\tau_{3}}\right\}$ is positively oriented in $\mathbb{H}_{\mathbb{R}}$.

Lemma 3 Let $R \in \mathbb{R}$ be a constant, then we have the following restriction formulas:

$$
\left.D x\right|_{\left\{X \in \mathbb{H}_{\mathbb{R}} ; N(X)=R\right\}}=\frac{X}{\|X\|} d S,\left.\quad D x\right|_{\left\{X \in \mathbb{H}_{\mathbb{R}} ;\|X\|=R\right\}}=\frac{X^{-}}{\|X\|} d S=\frac{X^{-}}{R} d S,
$$

where the sets $\left\{X \in \mathbb{H}_{\mathbb{R}} ; N(X)=R\right\}$ and $\left\{X \in \mathbb{H}_{\mathbb{R}} ;\|X\|=R\right\}$ are oriented as boundaries of the open sets $\left\{X \in \mathbb{H}_{\mathbb{R}} ; N(X)<R\right\}$ and $\left\{X \in \mathbb{H}_{\mathbb{R}} ;\|X\|<R\right\}$ respectively, and $d S$ denotes the respective restrictions of the Euclidean measure on $\mathbb{H}_{\mathbb{R}}$.

\section{$3 \quad$ Regular Functions on $\mathbb{H}$ and $\mathbb{H}_{\mathbb{C}}$}

Recall that regular functions on $\mathbb{H}$ are defined using an analogue of the Cauchy-Riemann equations. Write $\tilde{X} \in \mathbb{H}$ as $\tilde{X}=\tilde{x}^{0} e_{0}+\tilde{x}^{1} e_{1}+\tilde{x}^{2} e_{2}+\tilde{x}^{3} e_{3}, \tilde{x}^{0}, \tilde{x}^{1}, \tilde{x}^{2}, \tilde{x}^{3} \in \mathbb{R}$, and factor the four-dimensional Laplacian operator $\square$ on $\mathbb{H}$ as a product of two Dirac operators

$$
=\frac{\partial^{2}}{\left(\partial \tilde{x}^{0}\right)^{2}}+\frac{\partial^{2}}{\left(\partial \tilde{x}^{1}\right)^{2}}+\frac{\partial^{2}}{\left(\partial \tilde{x}^{2}\right)^{2}}+\frac{\partial^{2}}{\left(\partial \tilde{x}^{3}\right)^{2}}=\nabla \nabla^{+}=\nabla^{+} \nabla,
$$


where

$$
\begin{aligned}
\nabla^{+} & =e_{0} \frac{\partial}{\partial \tilde{x}^{0}}+e_{1} \frac{\partial}{\partial \tilde{x}^{1}}+e_{2} \frac{\partial}{\partial \tilde{x}^{2}}+e_{3} \frac{\partial}{\partial \tilde{x}^{3}} \quad \text { and } \\
\nabla & =e_{0} \frac{\partial}{\partial \tilde{x}^{0}}-e_{1} \frac{\partial}{\partial \tilde{x}^{1}}-e_{2} \frac{\partial}{\partial \tilde{x}^{2}}-e_{3} \frac{\partial}{\partial \tilde{x}^{3}} .
\end{aligned}
$$

The operators $\nabla^{+}, \nabla$ can be applied to functions on the left and on the right. For an open subset $U \subset \mathbb{H}$ and a differentiable function $f$ on $U$ with values in $\mathbb{H}$ or $\mathbb{H}_{\mathbb{C}}$, we say $f$ is left-regular if $\left(\nabla^{+} f\right)(\tilde{X})=0$ for all $\tilde{X} \in U$, and $f$ is right-regular if $\left(f \nabla^{+}\right)(\tilde{X})=0$ for all $\tilde{X} \in U$.

Proposition 4 For any $\mathcal{C}^{1}$-function $f$ on $U \subset \mathbb{H}$ with values in $\mathbb{H}$ or $\mathbb{H}_{\mathbb{C}}$,

$$
d(f \cdot D \tilde{x})=d f \wedge D \tilde{x}=\left.\left(f \nabla^{+}\right) d V\right|_{\mathbb{H}}, \quad d(D \tilde{x} \cdot f)=-D \tilde{x} \wedge d f=\left.\left(\nabla^{+} f\right) d V\right|_{\mathbb{H}^{-}}
$$

In particular,

$$
\nabla^{+} f=0 \quad \Longleftrightarrow \quad D \tilde{x} \wedge d f=0, \quad f \nabla^{+}=0 \quad \Longleftrightarrow \quad d f \wedge D \tilde{x}=0 .
$$

Following [FL1], we say that a differential function $f^{\mathbb{C}}: U^{\mathbb{C}} \rightarrow \mathbb{H}_{\mathbb{C}}$ defined on an open set $U^{\mathbb{C}} \subset \mathbb{H}_{\mathbb{C}}$ is holomorphic if it is holomorphic with respect to the complex variables $z^{0}, z^{1}, z^{2}, z^{3}$. Then we define $f^{\mathbb{C}}$ to be holomorphic left-regular if it is holomorphic and $\nabla^{+} f^{\mathbb{C}}=0$. Similarly, $f^{\mathbb{C}}$ is defined to be holomorphic right-regular if it is holomorphic and $f^{\mathbb{C}} \nabla^{+}=0$.

If we identify $\mathbb{H}_{\mathbb{C}}$ with complex $2 \times 2$ matrices $\left(\begin{array}{ll}z_{11} & z_{12} \\ z_{21} & z_{22}\end{array}\right), z_{i j} \in \mathbb{C}$, then a function $f^{\mathbb{C}}$ : $U^{\mathbb{C}} \rightarrow \mathbb{H}_{\mathbb{C}}$ is holomorphic if and only if it is holomorphic with respect to the complex variables $z_{i j}, 1 \leq i, j \leq 2$. Let us introduce holomorphic analogues of $\nabla^{+}$and $\nabla$ :

$$
\begin{aligned}
& \nabla_{\mathbb{C}}^{+}=e_{0} \frac{\partial}{\partial z^{0}}+e_{1} \frac{\partial}{\partial z^{1}}+e_{2} \frac{\partial}{\partial z^{2}}+e_{3} \frac{\partial}{\partial z^{3}} \quad \text { and } \\
& \nabla_{\mathbb{C}}=e_{0} \frac{\partial}{\partial z^{0}}-e_{1} \frac{\partial}{\partial z^{1}}-e_{2} \frac{\partial}{\partial z^{2}}-e_{3} \frac{\partial}{\partial z^{3}} .
\end{aligned}
$$

Then for a holomorphic function $f^{\mathbb{C}}: U^{\mathbb{C}} \rightarrow \mathbb{H}_{\mathbb{C}}$, the following derivatives are equal:

$$
\begin{gathered}
\nabla^{+} f^{\mathbb{C}}=\nabla_{\mathbb{C}}^{+} f^{\mathbb{C}}=2\left(\begin{array}{cc}
\frac{\partial}{\partial z_{22}} & -\frac{\partial}{\partial z_{21}} \\
-\frac{\partial}{\partial z_{12}} & \frac{\partial}{\partial z_{11}}
\end{array}\right) f^{\mathbb{C}}, \quad f^{\mathbb{C}} \nabla^{+}=f^{\mathbb{C}} \nabla_{\mathbb{C}}^{+}=2 f^{\mathbb{C}}\left(\begin{array}{cc}
\frac{\partial}{\partial z_{22}} & -\frac{\partial}{\partial z_{21}} \\
-\frac{\partial}{\partial z_{12}} & \frac{\partial}{\partial z_{11}}
\end{array}\right), \\
\nabla f^{\mathbb{C}}=\nabla_{\mathbb{C}} f^{\mathbb{C}}=2\left(\begin{array}{cc}
\frac{\partial}{\partial z_{11}} & \frac{\partial}{\partial z_{21}} \\
\frac{\partial}{\partial z_{12}} & \frac{\partial}{\partial z_{22}}
\end{array}\right) f^{\mathbb{C}}, \quad f^{\mathbb{C}} \nabla=f^{\mathbb{C}} \nabla_{\mathbb{C}}=2 f^{\mathbb{C}}\left(\begin{array}{cc}
\frac{\partial}{\partial z_{11}} & \frac{\partial}{\partial z_{21}} \\
\frac{\partial}{\partial z_{12}} & \frac{\partial}{\partial z_{22}}
\end{array}\right) .
\end{gathered}
$$

Proposition 5 For any holomorphic function $f^{\mathbb{C}}: U^{\mathbb{C}} \rightarrow \mathbb{H}_{\mathbb{C}}$,

$$
\nabla_{\mathbb{C}}^{+} f^{\mathbb{C}}=0 \quad \Longleftrightarrow \quad D z \wedge d f^{\mathbb{C}}=0, \quad f^{\mathbb{C}} \nabla_{\mathbb{C}}^{+}=0 \quad \Longleftrightarrow \quad d f^{\mathbb{C}} \wedge D z=0 .
$$

Lemma 6 We have:

1. $\square \frac{1}{N(Z)}=0$;

2. $\nabla_{\mathbb{C}} \frac{1}{N(Z)}=\frac{1}{N(Z)} \nabla_{\mathbb{C}}=-2 \frac{Z^{-1}}{N(Z)}=-2 \frac{Z^{+}}{N(Z)^{2}} ;$ 
3. $\frac{Z^{-1}}{N(Z)}=\frac{Z^{+}}{N(Z)^{2}}$ is a holomorphic left- and right-regular function defined wherever $N(Z) \neq 0$;

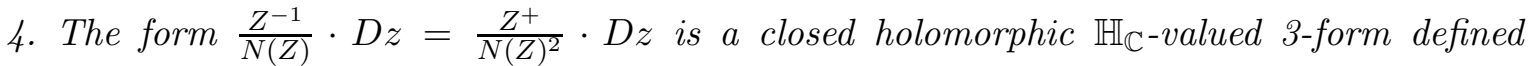
wherever $N(Z) \neq 0$.

Lemma 7 Let $U^{\mathbb{C}} \subset \mathbb{H}_{\mathbb{C}}$ be an open subset. For any differentiable function $F: U^{\mathbb{C}} \rightarrow \mathbb{C}$, we have:

$$
\begin{gathered}
\nabla\left(F\left(Z^{+}\right)\right)=\left(\nabla^{+} F\right)\left(Z^{+}\right), \quad \nabla^{+}\left(F\left(Z^{+}\right)\right)=(\nabla F)\left(Z^{+}\right), \\
\nabla\left(F\left(Z^{-1}\right)\right)=-Z^{-1} \cdot(\nabla F)\left(Z^{-1}\right) \cdot Z^{-1} .
\end{gathered}
$$

\section{Regular Functions on $\mathbb{H}_{\mathbb{R}}$}

We introduce linear differential operators on $\mathbb{H}_{\mathbb{R}}$

$$
\begin{aligned}
& \nabla_{\mathbb{R}}^{+}=e_{0} \frac{\partial}{\partial x^{0}}-\tilde{e}_{1} \frac{\partial}{\partial x^{1}}-\tilde{e}_{2} \frac{\partial}{\partial x^{2}}+e_{3} \frac{\partial}{\partial x^{3}} \quad \text { and } \\
& \nabla_{\mathbb{R}}=e_{0} \frac{\partial}{\partial x^{0}}+\tilde{e}_{1} \frac{\partial}{\partial x^{1}}+\tilde{e}_{2} \frac{\partial}{\partial x^{2}}-e_{3} \frac{\partial}{\partial x^{3}}
\end{aligned}
$$

which may be applied to functions on the left and on the right.

Fix an open subset $U \subset \mathbb{H}_{\mathbb{R}}$ and let $f$ be a differentiable function on $U$ with values in $\mathbb{H}_{\mathbb{R}}$ or $\mathbb{H}_{\mathbb{C}}$.

Definition 8 The function $f$ is left-regular if it satisfies

$$
\left(\nabla_{\mathbb{R}}^{+} f\right)(X)=e_{0} \frac{\partial f}{\partial x^{0}}(X)-\tilde{e}_{1} \frac{\partial f}{\partial x^{1}}(X)-\tilde{e}_{2} \frac{\partial f}{\partial x^{2}}(X)+e_{3} \frac{\partial f}{\partial x^{3}}(X)=0, \quad \forall X \in U .
$$

Similarly, $f$ is right-regular if

$$
\left(f \nabla_{\mathbb{R}}^{+}\right)(X)=\frac{\partial f}{\partial x^{0}}(X) e_{0}-\frac{\partial f}{\partial x^{1}}(X) \tilde{e}_{1}-\frac{\partial f}{\partial x^{2}}(X) \tilde{e}_{2}+\frac{\partial f}{\partial x^{3}}(X) e_{3}=0, \quad \forall X \in U .
$$

We denote by $\square_{2,2}$ the ultrahyperbolic wave operator on $\mathbb{H}_{\mathbb{R}}$ which can be factored as follows:

$$
\square_{2,2}=\frac{\partial^{2}}{\left(\partial x^{0}\right)^{2}}-\frac{\partial^{2}}{\left(\partial x^{1}\right)^{2}}-\frac{\partial^{2}}{\left(\partial x^{2}\right)^{2}}+\frac{\partial^{2}}{\left(\partial x^{3}\right)^{2}}=\nabla_{\mathbb{R}} \nabla_{\mathbb{R}}^{+}=\nabla_{\mathbb{R}}^{+} \nabla_{\mathbb{R}}
$$

Proposition 9 For any $\mathcal{C}^{1}$-function $f: U \rightarrow \mathbb{H}_{\mathbb{R}}\left(\right.$ or $\left.f: U \rightarrow \mathbb{H}_{\mathbb{C}}\right)$,

$$
d(f \cdot D x)=d f \wedge D x=\left(f \nabla_{\mathbb{R}}^{+}\right) d V, \quad d(D x \cdot f)=-D x \wedge d f=\left(\nabla_{\mathbb{R}}^{+} f\right) d V .
$$

In particular,

$$
\nabla_{\mathbb{R}}^{+} f=0 \quad \Longleftrightarrow \quad D x \wedge d f=0, \quad f \nabla_{\mathbb{R}}^{+}=0 \quad \Longleftrightarrow \quad d f \wedge D x=0 .
$$

Let $U^{\mathbb{C}} \subset \mathbb{H}_{\mathbb{C}}$ be an open set. The restriction relations

$$
\left.D z\right|_{\mathbb{H}_{\mathbb{R}}}=D x,\left.\quad D z\right|_{\mathbb{H}}=D \tilde{x}
$$

imply that the restriction of a holomorphic left- or right-regular function to $U^{\mathbb{R}}=U^{\mathbb{C}} \cap \mathbb{H}_{\mathbb{R}}$ produces a left- or right-regular function on $U^{\mathbb{R}}$ respectively. And the restriction of a holomorphic 
left- or right-regular function to $U_{\mathbb{H}}=U^{\mathbb{C}} \cap \mathbb{H}$ also produces a left- or right-regular function on $U_{\mathbb{H}}$ respectively. Conversely, if one starts with, say, a left-regular function on $\mathbb{H}_{\mathbb{R}}$, extends it holomorphically to a left-regular function on $\mathbb{H}_{\mathbb{C}}$ and then restricts the extension to $\mathbb{H}$, the resulting function is left-regular on $\mathbb{H}$.

These properties of Dirac operators $\nabla_{\mathbb{R}}^{+}$and $\nabla_{\mathbb{R}}$ and the notion of regular functions on $\mathbb{H}_{\mathbb{R}}$ are in complete parallel with the Dirac operators $\nabla_{\mathbb{M}}^{+}$and $\nabla_{\mathbb{M}}$ and the corresponding notion of regular functions on $\mathbb{M}$ introduced in Section 3.2 in [FL1].

Proposition 10 Let $f^{\mathbb{C}}: U^{\mathbb{C}} \rightarrow \mathbb{H}_{\mathbb{C}}$ be a holomorphic function. Then

$$
\begin{array}{cl}
\nabla^{+} f^{\mathbb{C}}=\nabla_{\mathbb{R}}^{+} f^{\mathbb{C}}=\nabla_{\mathbb{M}}^{+} f^{\mathbb{C}}=\nabla^{+} f^{\mathbb{C}}, \quad f^{\mathbb{C}} \nabla^{+}=f^{\mathbb{C}} \nabla_{\mathbb{R}}^{+}=f^{\mathbb{C}} \nabla_{\mathbb{M}}^{+}=f^{\mathbb{C}} \nabla^{+}, \\
\nabla f^{\mathbb{C}}=\nabla_{\mathbb{R}} f^{\mathbb{C}}=\nabla_{\mathbb{M}} f^{\mathbb{C}}=\nabla f^{\mathbb{C}}, \quad & f^{\mathbb{C}} \nabla=f^{\mathbb{C}} \nabla_{\mathbb{R}}=f^{\mathbb{C}} \nabla_{\mathbb{M}}=f^{\mathbb{C}} \nabla .
\end{array}
$$

Thus, essentially by design, the Dirac operators $\nabla^{+}, \nabla_{\mathbb{R}}^{+}, \nabla_{\mathbb{M}}^{+}, \nabla_{\mathbb{C}}^{+}$and $\nabla, \nabla_{\mathbb{R}}, \nabla_{\mathbb{M}}, \nabla_{\mathbb{C}}$ (and hence the notions of regular functions on $\mathbb{H}, \mathbb{H}_{\mathbb{R}}, \mathbb{M}$ and holomorphic regular functions on $\left.\mathbb{H}_{\mathbb{C}}\right)$ are all compatible.

\section{$5 \quad$ Fueter Formula for Holomorphic Regular Functions on $\mathbb{H}_{\mathbb{R}}$}

We are interested in extensions of the Cauchy-Fueter formula to functions on $\mathbb{H}_{\mathbb{R}}$. First we recall the classical version of the integral formula due to Fueter:

Theorem 11 (Cauchy-Fueter Formula [F1, F2]) Let $U_{\mathbb{H}} \subset \mathbb{H}$ be an open bounded subset with piecewise $\mathcal{C}^{1}$ boundary $\partial U_{\mathbb{H}}$. Suppose that $f(\tilde{X})$ is left-regular on a neighborhood of the closure $\overline{U_{\mathbb{H}}}$, then

$$
\frac{1}{2 \pi^{2}} \int_{\partial U_{\mathbb{H}}} \frac{\left(\tilde{X}-\tilde{X}_{0}\right)^{-1}}{N\left(\tilde{X}-\tilde{X}_{0}\right)} \cdot D \tilde{x} \cdot f(\tilde{X})= \begin{cases}f\left(\tilde{X}_{0}\right) & \text { if } \tilde{X}_{0} \in U_{\mathbb{H}} \\ 0 & \text { if } \tilde{X}_{0} \notin \overline{U_{\mathbb{H}}}\end{cases}
$$

If $g(\tilde{X})$ is right-regular on a neighborhood of the closure $\overline{U_{\mathbb{H}}}$, then

$$
\frac{1}{2 \pi^{2}} \int_{\partial U_{\mathbb{H}}} g(\tilde{X}) \cdot D \tilde{x} \cdot \frac{\left(\tilde{X}-\tilde{X}_{0}\right)^{-1}}{N\left(\tilde{X}-\tilde{X}_{0}\right)}= \begin{cases}g\left(\tilde{X}_{0}\right) & \text { if } \tilde{X}_{0} \in U_{\mathbb{H}} \\ 0 & \text { if } \tilde{X}_{0} \notin \overline{U_{\mathbb{H}}}\end{cases}
$$

Let $U \subset \mathbb{H}_{\mathbb{R}}$ be an open subset, and let $f$ be a $\mathcal{C}^{1}$-function defined on a neighborhood of $\bar{U}$ such that $\nabla_{\mathbb{R}}^{+} f=0$. In this subsection we extend the Cauchy-Fueter integral formula to leftregular functions which can be extended holomorphically to a neighborhood of $\bar{U}$ in $\mathbb{H}_{\mathbb{C}}$. (In other words, we assume that $f$ is a real-analytic function on $\bar{U}$.) Observe that the expression in the integral formula $\frac{\left(\tilde{X}-\tilde{X}_{0}\right)^{-1}}{N\left(\tilde{X}-\tilde{X}_{0}\right)} \cdot D \tilde{x}$ is nothing else but the restriction to $\mathbb{H}$ of the holomorphic 3 -form $\frac{\left(Z-\tilde{X}_{0}\right)^{-1}}{N\left(Z-\tilde{X}_{0}\right)} \cdot D z$ which is the form from Lemma 6 translated by $\tilde{X}_{0}$. For this reason we expect an integral formula of the kind

$$
f\left(X_{0}\right)=\left.\frac{1}{2 \pi^{2}} \int_{\partial U}\left(\frac{\left(Z-X_{0}\right)^{-1}}{N\left(Z-X_{0}\right)} \cdot D z\right)\right|_{\mathbb{H}_{\mathbb{R}}} \cdot f(X), \quad \forall X_{0} \in U
$$

However, the integrand is singular wherever $N\left(Z-X_{0}\right)=0$. We resolve this difficulty by deforming the contour of integration $\partial U$ in such a way that the integral is no longer singular.

Fix an $\varepsilon \in \mathbb{R}$ and define an $\varepsilon$-deformation $h_{\varepsilon}: \mathbb{H}_{\mathbb{C}} \rightarrow \mathbb{H}_{\mathbb{C}}, Z \mapsto Z_{\varepsilon}$, by: 


$$
\begin{array}{lllll}
z_{11} & \mapsto \quad z_{11}+i \varepsilon z_{11}, & z_{12} & \mapsto & z_{12}-i \varepsilon z_{12}, \\
z_{21} & \mapsto \quad z_{21}-i \varepsilon z_{21}, & z_{22} & \mapsto & z_{22}+i \varepsilon z_{22} .
\end{array}
$$

Define a quadratic form on $\mathbb{H}_{\mathbb{C}}$

$$
S(Z)=z_{11} z_{22}+z_{12} z_{21}
$$

Lemma 12 We have the following identities:

$$
\begin{gathered}
Z_{\varepsilon}=Z+i \varepsilon Z^{-}, \quad\left(Z_{\varepsilon}\right)^{+}=Z^{+}+i \varepsilon Z^{+-}, \\
N\left(Z_{\varepsilon}\right)=\left(1-\varepsilon^{2}\right) N(Z)+2 i \varepsilon S(Z), \quad S(X)=\|X\|^{2}, \quad \forall X \in \mathbb{H}_{\mathbb{R}} .
\end{gathered}
$$

For $Z_{0} \in \mathbb{H}_{\mathbb{C}}$ fixed, we use a notation

$$
h_{\varepsilon, Z_{0}}(Z)=Z_{0}+h_{\varepsilon}\left(Z-Z_{0}\right)=Z+i \varepsilon\left(Z-Z_{0}\right)^{-}
$$

Theorem 13 Let $U \subset \mathbb{H}_{\mathbb{R}}$ be an open bounded subset with piecewise $\mathcal{C}^{1}$ boundary $\partial U$, and let $f(X)$ be a $\mathcal{C}^{1}$-function defined on a neighborhood of the closure $\bar{U}$ such that $\nabla_{\mathbb{R}}^{+} f=0$. Suppose that $f$ extends to a holomorphic left-regular function $f^{\mathbb{C}}: V^{\mathbb{C}} \rightarrow \mathbb{H}_{\mathbb{C}}$ with $V^{\mathbb{C}} \subset \mathbb{H}_{\mathbb{C}}$ an open subset containing $\bar{U}$, then

$$
-\frac{1}{2 \pi^{2}} \int_{\left(h_{\varepsilon, X_{0}}\right)_{*}(\partial U)} \frac{\left(Z-X_{0}\right)^{-1}}{N\left(Z-X_{0}\right)} \cdot D z \cdot f^{\mathbb{C}}(Z)= \begin{cases}f\left(X_{0}\right) & \text { if } X_{0} \in U ; \\ 0 & \text { if } X_{0} \notin \bar{U},\end{cases}
$$

for all $\varepsilon \neq 0$ sufficiently close to 0 .

Remark 14 For all $\varepsilon \neq 0$ sufficiently close to 0 the contour of integration $\left(h_{\varepsilon, X_{0}}\right)_{*}(\partial U)$ lies inside $V^{\mathbb{C}}$ and the integrand is non-singular, thus the integrals are well-defined. Moreover, we will see that the value of the integral becomes constant when the parameter $\varepsilon$ is sufficiently close to 0 . Of course, there is a similar formula for right-regular functions on $\mathbb{H}_{\mathbb{R}}$ as well.

Proof. Let $M=\sup _{X \in \partial U}\left\|X-X_{0}\right\|$. Without loss of generality we may assume that $V^{\mathbb{C}}$ is the $\delta$-neighborhood of $\bar{U}$ for some $\delta>0$. We will show that the integral formula holds for $0<|\varepsilon|<\delta / M$. Clearly, for this choice of $\varepsilon$ the contour of integration $\left(h_{\varepsilon, X_{0}}\right)_{*}(\partial U)$ lies inside $V^{\mathbb{C}}$ and, since the integrand is a closed form, the integral stays constant for $-\delta / M<\varepsilon<0$ and $0<\varepsilon<\delta / M$ (a priori the values of the integral may be different on these two intervals).

Let $S_{r}=\left\{X \in \mathbb{H}_{\mathbb{R}} ;\left\|X-X_{0}\right\|^{2}=r^{2}\right\}$ and $B_{r}=\left\{X \in \mathbb{H}_{\mathbb{R}} ;\left\|X-X_{0}\right\|^{2} \leq r^{2}\right\}$ be the sphere and the closed ball of radius $r$ centered at $X_{0}$, and choose $r>0$ sufficiently small so that $B_{r} \subset U$ and $r<\delta$.

Lemma 15 Let $\tilde{S}_{r}=\left\{\tilde{X} \in \mathbb{H}+X_{0} ;\left\|\tilde{X}-X_{0}\right\|^{2}=r^{2}\right\}$ be the sphere oriented as the boundary of the open ball, then

$$
\left(h_{\varepsilon, X_{0}}\right)_{*}(\partial U) \sim \begin{cases}-\tilde{S}_{r} & \text { if } X_{0} \in U ; \\ 0 & \text { if } X_{0} \notin \bar{U}\end{cases}
$$

as homology 3-cycles inside $\left\{Z \in V^{\mathbb{C}} ; N\left(Z-X_{0}\right) \neq 0\right\}$.

Proof. We give a proof for $\varepsilon>0$; the case $\varepsilon<0$ is similar. As homology cycles in $\{Z \in$ $\left.V^{\mathbb{C}} ; N\left(Z-X_{0}\right) \neq 0\right\}$

$$
\left(h_{\varepsilon, X_{0}}\right)_{*}(\partial U) \sim\left(h_{\varepsilon, X_{0}}\right)_{*}\left(S_{r}\right) \sim\left(h_{1, X_{0}}\right)_{*}\left(S_{r}\right) .
$$


If $X_{0} \notin \bar{U}$, the cycle $\left(h_{1, X_{0}}\right)_{*}\left(S_{r}\right)$ is homologous to zero.

Assume now $X_{0} \in U$. Let $\mathrm{P}_{\mathbb{H}}$ be the projection $\mathbb{H}_{\mathbb{C}} \rightarrow \mathbb{H}$ defined by

$$
\begin{aligned}
Z=\left(\tilde{x}^{0}+i \tilde{y}^{0}\right) e_{0}+ & \left(\tilde{x}^{1}+i \tilde{y}^{1}\right) e_{1}+\left(\tilde{x}^{2}+i \tilde{y}^{2}\right) e_{2}+\left(\tilde{x}^{3}+i \tilde{y}^{3}\right) e_{3} \\
& \mapsto \tilde{X}=\tilde{x}^{0} e_{0}+\tilde{x}^{1} e_{1}+\tilde{x}^{2} e_{2}+\tilde{x}^{3} e_{3}, \quad \tilde{x}^{0}, \tilde{x}^{1}, \tilde{x}^{2}, \tilde{x}^{3}, \tilde{y}^{0}, \tilde{y}^{1}, \tilde{y}^{2}, \tilde{y}^{3} \in \mathbb{R}
\end{aligned}
$$

and let $\mathrm{P}_{\mathbb{H}+X_{0}}: \mathbb{H}_{\mathbb{C}} \rightarrow \mathbb{H}+X_{0}$, be the projection $\mathrm{P}_{\mathbb{H}+X_{0}}(Z)=\mathrm{P}_{\mathbb{H}}\left(Z-X_{0}\right)+X_{0}$. We describe the supports of the cycles involved:

$$
\begin{aligned}
\left|S_{r}\right| & =\left\{X_{0}+a e_{0}+b \tilde{e}_{1}+c \tilde{e}_{2}+d e_{3} ; a^{2}+b^{2}+c^{2}+d^{2}=r^{2}\right\} \\
\left|\left(h_{1, X_{0}}\right)_{*}\left(S_{r}\right)\right|=\left\{X_{0}+(1+i) a e_{0}+(1-i) b \tilde{e}_{1}+(1-i) c \tilde{e}_{2}+(1+i) d e_{3} ;\right. & \left.a^{2}+b^{2}+c^{2}+d^{2}=r^{2}\right\} \\
\left|\left(\mathrm{P}_{\mathbb{H}+X_{0}} \circ h_{1, X_{0}}\right)_{*}\left(S_{r}\right)\right| & =\left\{X_{0}+a e_{0}+b e_{1}-c e_{2}+d e_{3} ; a^{2}+b^{2}+c^{2}+d^{2}=r^{2}\right\}=\left|\tilde{S}_{r}\right| .
\end{aligned}
$$

Moreover,

$$
\left(\mathrm{P}_{\mathbb{H}+X_{0}} \circ h_{1, X_{0}}\right)_{*}\left(S_{r}\right)=-\tilde{S}_{r}
$$

as homology cycles. It is easy to see that this projection provides a homotopy between $\left(h_{1, X_{0}}\right)_{*}\left(S_{r}\right)$ and $-S_{r}$, hence the lemma.

By Stokes'

$$
\int_{\left(h_{\varepsilon, X_{0}}\right)_{*}(\partial U)} \frac{\left(Z-X_{0}\right)^{-1}}{N\left(Z-X_{0}\right)} \cdot D z \cdot f^{\mathbb{C}}(Z)=\int_{-\tilde{S}_{r}} \frac{\left(Z-X_{0}\right)^{-1}}{N\left(Z-X_{0}\right)} \cdot D z \cdot f^{\mathbb{C}}(Z) \quad \text { if } X_{0} \in U,
$$

and zero if $X_{0} \notin \bar{U}$. Finally, by the Fueter formula for the usual quaternions (Theorem 11), the last integral is $-2 \pi^{2} f\left(X_{0}\right)$. (Alternatively, one can let $r \rightarrow 0^{+}$and show directly that the integral remains unchanged and at the same time approaches $-2 \pi^{2} f\left(X_{0}\right)$ in the same way the Cauchy and Cauchy-Fueter formulas are proved.)

For a Cauchy-Fueter formula for regular functions on $\mathbb{M}$ that extend to holomorphic regular functions on $\mathbb{H}_{\mathbb{C}}$ see Section 3.3 in [FL1].

\section{$6 \quad$ Fueter Formula for Regular Functions on $\mathbb{H}_{\mathbb{R}}$}

In this section we prove an analogue of the Cauchy-Fueter formula for smooth left-regular functions on $\mathbb{H}_{\mathbb{R}}$ which are not necessarily real analytic and do not necessarily have holomorphic extensions. As a "trade-off" for working with "bad" functions the proofs become much more involved.

Theorem 16 Let $U \subset \mathbb{H}_{\mathbb{R}}$ be a bounded open region with smooth boundary $\partial U$. Let $f: U \rightarrow \mathbb{H}_{\mathbb{C}}$ be a function which extends to a real-differentiable function on an open neighborhood $V \subset \mathbb{H}_{\mathbb{R}}$ of the closure $\bar{U}$ such that $\nabla_{\mathbb{R}}^{+} f=0$. Then, for any point $X_{0} \in \mathbb{H}_{\mathbb{R}}$ such that $\partial U$ intersects the cone $\left\{X \in \mathbb{H}_{\mathbb{R}} ; N\left(X-X_{0}\right)=0\right\}$ transversally, we have:

$$
\lim _{\varepsilon \rightarrow 0} \frac{-1}{2 \pi^{2}} \int_{\partial U} \frac{\left(X-X_{0}\right)^{+}}{\left(N\left(X-X_{0}\right)+i \varepsilon\left\|X-X_{0}\right\|^{2}\right)^{2}} \cdot D z \cdot f(X)= \begin{cases}f\left(X_{0}\right) & \text { if } X_{0} \in U \\ 0 & \text { if } X_{0} \notin \bar{U}\end{cases}
$$


Proof. The case $X_{0} \notin \bar{U}$ is easier, so we assume $X_{0} \in U$. Using Proposition 9, we get

$$
\begin{aligned}
d\left(\frac{\left(X-X_{0}\right)^{+}}{\left(N\left(X-X_{0}\right)+i \varepsilon\left\|X-X_{0}\right\|^{2}\right)^{2}} \cdot D z \cdot f(X)\right) \\
=\left(\frac{\left(X-X_{0}\right)^{+}}{\left(N\left(X-X_{0}\right)+i \varepsilon\left\|X-X_{0}\right\|^{2}\right)^{2}} \nabla_{\mathbb{R}}^{+}\right) f(X) d V \\
=4 i \varepsilon \frac{\left\|X-X_{0}\right\|^{2}-\left(X-X_{0}\right)^{+}\left(X-X_{0}\right)^{-}}{\left(N\left(X-X_{0}\right)+i \varepsilon\left\|X-X_{0}\right\|^{2}\right)^{3}} f(X) d V .
\end{aligned}
$$

In particular, expression (5) tends to zero pointwise when $\varepsilon \rightarrow 0$ except for those $X$ which lie on the cone $\left\{X \in \mathbb{H}_{\mathbb{R}} ; N\left(X-X_{0}\right)=0\right\}$, and we need to be very careful there. By translation we can assume that $X_{0}=0$. Let $S_{r}=\left\{X \in \mathbb{H}_{\mathbb{R}} ;\|X\|^{2}=r^{2}\right\}$ and $B_{r}=\left\{X \in \mathbb{H}_{\mathbb{R}} ;\|X\|^{2} \leq r^{2}\right\}$ be the sphere and the closed ball of radius $r$. By Stokes'

$$
\begin{array}{r}
\int_{\partial U} \frac{X^{+}}{\left(N(X)+i \varepsilon\|X\|^{2}\right)^{2}} \cdot D z \cdot f(X)=\int_{U \backslash B_{r}} d\left(\frac{X^{+}}{\left(N(X)+i \varepsilon\|X\|^{2}\right)^{2}} \cdot D z \cdot f(X)\right) \\
+\int_{S_{r}} \frac{X^{+}}{\left(N(X)+i \varepsilon r^{2}\right)^{2}} \cdot D z \cdot f(X) .
\end{array}
$$

We will show that, as $\varepsilon \rightarrow 0$, the first integral on the right hand side tends to zero (this part is non-trivial and uses that the cone $\left\{N\left(X-X_{0}\right)=0\right\}$ intersects $\partial U$ transversally). On the other hand, as $\varepsilon \rightarrow 0$ and $r \rightarrow 0^{+}$, the second integral tends to $-2 \pi^{2} f(0)$. The proof is essentially a series of integration by parts computations. In regular coordinates $\left(x^{0}, x^{1}, x^{2}, x^{3}\right)$ we have $X=x^{0} e_{0}+x^{1} \tilde{e}_{1}+x^{2} \tilde{e}_{2}+x^{3} e_{3}$, and $N(X)=\left(x^{0}\right)^{2}-\left(x^{1}\right)^{2}-\left(x^{2}\right)^{2}+\left(x^{3}\right)^{2}$. For computing purposes we replace $\left(x^{0}, x^{1}, x^{2}, x^{3}\right)$ with spherical coordinates $(\rho, \theta, \varphi, \psi)$ so that

$$
\begin{array}{lc}
x^{0}=\rho \cos \theta \cos \varphi & \rho \geq 0, \\
x^{1}=\rho \sin \theta \sin \psi & 0 \leq \theta \leq \pi / 2, \\
x^{2}=\rho \sin \theta \cos \psi & 0 \leq \varphi \leq 2 \pi, \\
x^{3}=\rho \cos \theta \sin \varphi & 0 \leq \psi \leq 2 \pi .
\end{array}
$$

This is an orientation-preserving change of coordinates, and the vector fields $\left\{\frac{\partial}{\partial \theta}, \frac{\partial}{\partial \varphi}, \frac{\partial}{\partial \psi}\right\}$ form a positively-oriented frame on $S_{r}$. Then

$$
N(X)=\rho^{2} \cos (2 \theta), \quad N(X)+i \varepsilon\|X\|^{2}=\rho^{2}(\cos (2 \theta)+i \varepsilon),
$$

and the equation $N(X)=0$ becomes $\theta=\pi / 4$.

Recall that the function $\frac{1}{x^{n}}$ which is singular at $x=0$ can be regularized as a distribution in two different ways, $\frac{1}{(x+i 0)^{n}}$ and $\frac{1}{(x-i 0)^{n}}$, so that a test function $g(x)$ is being sent into $\left\langle\frac{1}{(x+i 0)^{n}}, g(x)\right\rangle=\lim _{\varepsilon \rightarrow 0^{+}} \int \frac{g(x) d x}{(x+i \varepsilon)^{n}}$ or $\left\langle\frac{1}{(x-i 0)^{n}}, g(x)\right\rangle=\lim _{\varepsilon \rightarrow 0^{-}} \int \frac{g(x) d x}{(x+i \varepsilon)^{n}}$. By a similar fashion we have the following lemma:

Lemma 17 Fix a $\theta_{0} \in\left(0, \frac{\pi}{4}\right)$, and let $n$ be a positive integer, then we have two distributions which send a test function $g(\theta)$ into the limits

$$
\lim _{\varepsilon \rightarrow 0^{+}} \int_{\frac{\pi}{4}-\theta_{0}}^{\frac{\pi}{4}+\theta_{0}} \frac{g(\theta) d \theta}{(\cos (2 \theta)+i \varepsilon)^{n}} \quad \text { and } \quad \lim _{\varepsilon \rightarrow 0^{-}} \int_{\frac{\pi}{4}-\theta_{0}}^{\frac{\pi}{4}+\theta_{0}} \frac{g(\theta) d \theta}{(\cos (2 \theta)+i \varepsilon)^{n}}
$$


Proof. We need to show that the limits exist and depend continuously on the test function $g(\theta)$. We do it by induction on $n$ using integration by parts. If $n=1$,

$$
\begin{aligned}
\int_{\frac{\pi}{4}-\theta_{0}}^{\frac{\pi}{4}+\theta_{0}} & \frac{g(\theta) d \theta}{\cos (2 \theta)+i \varepsilon}=\int_{\frac{\pi}{4}-\theta_{0}}^{\frac{\pi}{4}+\theta_{0}} \frac{2 \sin (2 \theta)}{\cos (2 \theta)+i \varepsilon} \cdot \frac{g(\theta)}{2 \sin (2 \theta)} d \theta \\
& =\int_{\frac{\pi}{4}-\theta_{0}}^{\frac{\pi}{4}+\theta_{0}} \log (\cos (2 \theta)+i \varepsilon) \cdot \frac{d}{d \theta}\left(\frac{g(\theta)}{2 \sin (2 \theta)}\right) d \theta-\left.\log (\cos (2 \theta)+i \varepsilon) \cdot \frac{g(\theta)}{2 \sin (2 \theta)}\right|_{\frac{\pi}{4}-\theta_{0}} ^{\frac{\pi}{4}+\theta_{0}} .
\end{aligned}
$$

For the purpose of this integration, the complex logarithm function is defined on the complex plane $\mathbb{C}$ minus the negative real axis, and the values of the logarithm lie in the strip $\{z \in$ $\mathbb{C} ;-\pi<\operatorname{Im} z<\pi\}$. The function $\log (\cos (2 \theta)+i \varepsilon)$ is integrable for all values of $\varepsilon$, including $\varepsilon=0$, hence the limits as $\varepsilon \rightarrow 0^{ \pm}$exist and depend continuously on $g(\theta)$.

Now suppose that $n>1$, then

$$
\begin{aligned}
\int_{\frac{\pi}{4}-\theta_{0}}^{\frac{\pi}{4}+\theta_{0}} \frac{g(\theta) d \theta}{(\cos (2 \theta)+i \varepsilon)^{n}=} & \int_{\frac{\pi}{4}-\theta_{0}}^{\frac{\pi}{4}+\theta_{0}} \frac{2 \sin (2 \theta)}{(\cos (2 \theta)+i \varepsilon)^{n}} \cdot \frac{g(\theta)}{2 \sin (2 \theta)} d \theta \\
= & \left.\frac{1}{n-1} \frac{1}{(\cos (2 \theta)+i \varepsilon)^{n-1}} \cdot \frac{g(\theta)}{2 \sin (2 \theta)}\right|_{\frac{\pi}{4}-\theta_{0}} ^{\frac{\pi}{4}+\theta_{0}} \\
& \quad-\frac{1}{n-1} \int_{\frac{\pi}{4}-\theta_{0}}^{\frac{\pi}{4}+\theta_{0}} \frac{1}{(\cos (2 \theta)+i \varepsilon)^{n-1}} \cdot \frac{d}{d \theta}\left(\frac{g(\theta)}{2 \sin (2 \theta)}\right) d \theta
\end{aligned}
$$

and the result follows by induction in $n$.

Lemma 18

$$
\lim _{\varepsilon \rightarrow 0} \int_{U \backslash B_{r}} d\left(\frac{X^{+}}{\left(N(X)+i \varepsilon\|X\|^{2}\right)^{2}} \cdot D z \cdot f(X)\right)=0 .
$$

Proof. We have seen that

$$
\begin{aligned}
\int_{U \backslash B_{r}} d\left(\frac{X^{+}}{\left(N(X)+i \varepsilon\|X\|^{2}\right)^{2}} \cdot D z \cdot f(X)\right) & \\
& =4 i \varepsilon \int_{U \backslash B_{r}} \frac{\left\|X-X_{0}\right\|^{2}-\left(X-X_{0}\right)^{+}\left(X-X_{0}\right)^{-}}{\left(N(X)+i \varepsilon\|X\|^{2}\right)^{3}} f(X) d V .
\end{aligned}
$$

Writing the right hand side integral in the spherical coordinates (6) and integrating out the variables $\rho, \varphi, \psi$ we obtain an integral of the type

$$
4 i \varepsilon \int_{0}^{\frac{\pi}{2}} \frac{g(\theta) d \theta}{(\cos (2 \theta)+i \varepsilon)^{3}},
$$

for some function $g(\theta)$. By assumption, the boundary $\partial U$ is smooth, compact and intersects the cone $\{N(X)=0\}=\{\theta=\pi / 4\}$ transversally, hence the function $g(\theta)$ is smooth at least for $\theta$ lying in some interval $\left[\frac{\pi}{4}-\theta_{0}, \frac{\pi}{4}+\theta_{0}\right]$ with $\theta_{0} \in\left(0, \frac{\pi}{4}\right)$. It follows from Lemma 17 that the limit of (7) as $\varepsilon \rightarrow 0$ is zero.

\section{Lemma 19}

$$
\int_{S_{r}} \frac{X^{+}}{\left(N(X)+i \varepsilon r^{2}\right)^{2}} \cdot D z=r \int_{S_{r}} \frac{d S}{\left(N(X)+i \varepsilon r^{2}\right)^{2}}=-\frac{2 \pi^{2}}{1+\varepsilon^{2}}
$$


Proof. From Lemma 3 we see that

$$
\left.\frac{X^{+}}{\left(N(X)+i \varepsilon r^{2}\right)^{2}} \cdot D z\right|_{S_{r}}=\frac{X^{+} X^{-}}{\left(N(X)+i \varepsilon r^{2}\right)^{2}} \cdot \frac{d S}{r} .
$$

Notice that the involution $X \mapsto X^{-}$preserves the sphere, its orientation, its volume form $d S$ and replaces $\frac{X^{+} X^{-}}{\left(N(X)+i \varepsilon r^{2}\right)^{2}}$ with $\frac{X^{+-} X}{\left(N(X)+i \varepsilon r^{2}\right)^{2}}$. Therefore, using $X^{+} X^{-}+X^{+-} X=2\|X\|^{2}$, spherical coordinates (6) and $d S=r^{3} \sin \theta \cos \theta d \psi d \varphi d \theta$,

$$
\begin{gathered}
\int_{S_{r}} \frac{X^{+} X^{-}}{\left(N(X)+i \varepsilon r^{2}\right)^{2}} \cdot \frac{d S}{r}=\frac{1}{2} \int_{S_{r}} \frac{X^{+} X^{-}+X^{+-} X}{\left(N(X)+i \varepsilon r^{2}\right)^{2}} \cdot \frac{d S}{r}=\int_{S_{r}} \frac{r d S}{\left(N(X)+i \varepsilon r^{2}\right)^{2}} \\
=\int_{\theta=0}^{\theta=\pi / 2} \int_{\varphi=0}^{\varphi=2 \pi} \int_{\psi=0}^{\psi=2 \pi} \frac{\sin \theta \cos \theta d \psi d \varphi d \theta}{(\cos (2 \theta)+i \varepsilon)^{2}}=2 \pi^{2} \int_{\theta=0}^{\theta=\pi / 2} \frac{\sin (2 \theta) d \theta}{(\cos (2 \theta)+i \varepsilon)^{2}} \\
=\left.\frac{\pi^{2}}{\cos (2 \theta)+i \varepsilon}\right|_{\theta=0} ^{\theta=\pi / 2}=\frac{\pi^{2}}{-1+i \varepsilon}-\frac{\pi^{2}}{1+i \varepsilon}=-\frac{2 \pi^{2}}{1+\varepsilon^{2}} .
\end{gathered}
$$

Lemma 20

$$
\lim _{r \rightarrow 0^{+}}\left(\lim _{\varepsilon \rightarrow 0} \int_{S_{r}} \frac{X^{+} \cdot D z \cdot f(X)}{\left(N(X)+i \varepsilon r^{2}\right)^{2}}\right)=-2 \pi^{2} f(0) .
$$

Proof. By Lemma 3 we have:

$$
\lim _{\varepsilon \rightarrow 0} \int_{S_{r}} \frac{X^{+} \cdot D z \cdot f(X)}{\left(N(X)+i \varepsilon r^{2}\right)^{2}}=\lim _{\varepsilon \rightarrow 0} \int_{S_{r}} \frac{X^{+} X^{-} \cdot f(X)}{\left(N(X)+i \varepsilon r^{2}\right)^{2}} \frac{d S}{r}
$$

If the function $f(X)$ were constant we would be done by previous lemma. However, we cannot argue that since $r \rightarrow 0^{+}, f(X)$ is close to $f(0)$ and so may be treated like a constant because there can be derivatives of $f(X)$ involved. So an integration by parts argument will be needed.

First we compute (writing $X=x^{0} e_{0}+x^{1} \tilde{e}_{1}+x^{2} \tilde{e}_{2}+x^{3} e_{3}$ )

$$
X^{+} X^{-}=\left(\begin{array}{cc}
\left(x^{0}\right)^{2}+\left(x^{1}\right)^{2}+\left(x^{2}\right)^{2}+\left(x^{3}\right)^{2} & 2\left(x^{2} x^{3}-x^{0} x^{1}\right)-2 i\left(x^{0} x^{2}+x^{1} x^{3}\right) \\
2\left(x^{2} x^{3}-x^{0} x^{1}\right)+2 i\left(x^{0} x^{2}+x^{1} x^{3}\right) & \left(x^{0}\right)^{2}+\left(x^{1}\right)^{2}+\left(x^{2}\right)^{2}+\left(x^{3}\right)^{2}
\end{array}\right),
$$

and using the spherical coordinates (6) we can rewrite

$$
X^{+} X^{-}=\rho^{2}\left(\begin{array}{ll}
1 & 0 \\
0 & 1
\end{array}\right)+\rho^{2} \sin (2 \theta) \sin (\psi-\varphi)\left(\begin{array}{ll}
0 & 1 \\
1 & 0
\end{array}\right)+\rho^{2} \sin (2 \theta) \cos (\psi-\varphi)\left(\begin{array}{cc}
0 & -i \\
i & 0
\end{array}\right) .
$$

Thus (8) can be rewritten as

$$
\lim _{\varepsilon \rightarrow 0}\left(r \int_{S_{r}} \frac{f(X) d S}{\left(N(X)+i \varepsilon r^{2}\right)^{2}}+r \int_{S_{r}} \frac{\sin (\psi-\varphi)\left(\begin{array}{cc}
0 & 1 \\
1 & 0
\end{array}\right)+\cos (\psi-\varphi)\left(\begin{array}{cc}
0 & -i \\
i & 0
\end{array}\right)}{\left(N(X)+i \varepsilon r^{2}\right)^{2}} \cdot \sin (2 \theta) f d S\right) .
$$

We have:

$$
\begin{aligned}
r \int_{S_{r}} & \frac{f(X) d S}{\left(N(X)+i \varepsilon r^{2}\right)^{2}}=\frac{1}{2} \int_{\theta=0}^{\theta=\pi / 2} \int_{\varphi=0}^{\varphi=2 \pi} \int_{\psi=0}^{\psi=2 \pi} \frac{\sin (2 \theta) f d \psi d \varphi d \theta}{(\cos (2 \theta)+i \varepsilon)^{2}} \\
\quad & =\frac{1}{4}\left(\left.\int_{\varphi=0}^{\varphi=2 \pi} \int_{\psi=0}^{\psi=2 \pi} \frac{f}{\cos (2 \theta)+i \varepsilon}\right|_{\theta=0} ^{\theta=\pi / 2} d \psi d \varphi-\int_{\theta=0}^{\theta=\pi / 2} \int_{\varphi=0}^{\varphi=2 \pi} \int_{\psi=0}^{\psi=2 \pi} \frac{\frac{\partial f}{\partial \theta} d \psi d \varphi d \theta}{\cos (2 \theta)+i \varepsilon}\right) .
\end{aligned}
$$


By the chain rule $\frac{\partial f}{\partial \theta}$ can be written as $\rho \cdot g(X)$ for some smooth function $g(X)$, thus the second integral is $r$ times an expression from Lemma 17. Taking limits $\varepsilon \rightarrow 0$ and then $r \rightarrow 0^{+}$, the first integral tends to $-2 \pi^{2} f(0)$ and the second tends to zero. The second term inside the limit is

$$
\begin{aligned}
& r \int_{S_{r}} \frac{\sin (\psi-\varphi)\left(\begin{array}{ll}
0 & 1 \\
1 & 0
\end{array}\right)+\cos (\psi-\varphi)\left(\begin{array}{cc}
0 & -i \\
i & 0
\end{array}\right)}{\left(N(X)+i \varepsilon r^{2}\right)^{2}} \cdot \sin (2 \theta) f d S \\
& \quad=\frac{1}{2} \int_{\theta=0}^{\theta=\pi / 2} \int_{\varphi=0}^{\varphi=2 \pi} \int_{\psi=0}^{\psi=2 \pi} \frac{\sin (\psi-\varphi)\left(\begin{array}{ll}
0 & 1 \\
1 & 0
\end{array}\right)+\cos (\psi-\varphi)\left(\begin{array}{cc}
0 & -i \\
i & 0
\end{array}\right)}{(\cos (2 \theta)+i \varepsilon)^{2}} \cdot \sin ^{2}(2 \theta) f d \psi d \varphi d \theta .
\end{aligned}
$$

But

$$
\begin{aligned}
& \int_{\psi=0}^{\psi=2 \pi} \sin (\psi-\varphi) f d \psi=\int_{\psi=0}^{\psi=2 \pi} \cos (\psi-\varphi) \frac{\partial f}{\partial \psi} f d \psi \\
& \int_{\psi=0}^{\psi=2 \pi} \cos (\psi-\varphi) f d \psi=-\int_{\psi=0}^{\psi=2 \pi} \sin (\psi-\varphi) \frac{\partial f}{\partial \psi} f d \psi
\end{aligned}
$$

By the chain rule $\frac{\partial f}{\partial \psi}$ can be written as $\rho \cdot h(X)$ for some smooth function $h(X)$, thus the right hand side of (9) is $r$ times an expression from Lemma 17. When we take limits first as $\varepsilon \rightarrow 0$ and then as $r \rightarrow 0^{+}$, integral (9) tends to zero.

This concludes our proof of Theorem [16.

\section{$7 \quad$ Separation of the Series for $S L(2, \mathbb{R})$}

What makes the representation theory of $S L(2, \mathbb{R})$ more interesting than that of $S U(2)$ is having the separation of the series into discrete and continuous components. Instead of $S L(2, \mathbb{R})$ we prefer to work with $S U(1,1)$ sitting inside $\mathbb{H}_{\mathbb{R}}$, as in (3). In this section we outline a relationship between split quaternionic analysis and the decomposition

$$
L^{2}(S U(1,1)) \simeq L_{\text {discr }}^{2}(S U(1,1)) \oplus L_{\text {cont }}^{2}(S U(1,1))
$$

The denominator of the Cauchy-Fueter kernel $N\left(X-X_{0}\right)$ determines a region in $\mathbb{H}_{\mathbb{C}}$

$$
\Omega=\left\{X_{0} \in \mathbb{H}_{\mathbb{C}} ; N\left(X-X_{0}\right) \neq 0 \quad \forall X \in \mathbb{H}_{\mathbb{R}} \text { with } N(X)=1\right\}
$$

This region contains two open Ol'shanskii semigroups of $G L(2, \mathbb{C})$

$$
\begin{gathered}
\Gamma^{0}=\left\{Z \in \mathbb{H}_{\mathbb{C}} ; Z^{*} \tilde{e}_{3} Z-\tilde{e}_{3} \text { is positive definite }\right\} \\
\overline{\Gamma^{0}}=\left(\Gamma^{0}\right)^{-1}=\left\{Z \in \mathbb{H}_{\mathbb{C}} ; Z^{*} \tilde{e}_{3} Z-\tilde{e}_{3} \text { is negative definite }\right\}
\end{gathered}
$$

where $\tilde{e}_{3}=i e_{3}=\left(\begin{array}{cc}1 & 0 \\ 0 & -1\end{array}\right) \in \mathbb{H}_{\mathbb{C}}$. Following $\left[\underline{\mathrm{Ku} \emptyset}\right.$ we can show that over $\Gamma^{0}$ and $\overline{\Gamma^{0}}$ the Cauchy-Fueter kernel $\frac{\left(Z-X_{0}\right)^{-1}}{N\left(Z-X_{0}\right)}$ can be expanded in terms of the $K$-types of the discrete series 
of $S U(1,1)$. Thus we obtain the following integral formula for the projector onto the discrete series of $S U(1,1))$ :

$$
\left(\mathrm{P}^{\text {discr }}\right) f\left(X_{0}\right)=\frac{1}{2 \pi^{2}} \int_{C_{d i s c r}} \frac{\left(Z-X_{0}\right)^{-1}}{N\left(Z-X_{0}\right)} \cdot D z \cdot f(Z)
$$

where $C_{d i s c r}$ is a certain three cycle in $\Gamma^{0} \cup \overline{\Gamma^{0}}$. (Strictly speaking, this operator is not a projector because it has eigenvalues \pm 1 on the discrete series subspace, but its square does give a projection onto the discrete series.) Furthermore, we can decompose the cycle $C_{d i s c r}$ into a sum of two cycles $C_{\text {discr }}^{+}+C_{\text {discr }}^{-}$with $C_{\text {discr }}^{+}$and $C_{\text {discr }}^{-}$lying in $\overline{\Gamma^{0}}$ and $\Gamma^{0}$ respectively. Then integration over $C_{d i s c r}^{+}$(respectively $C_{d i s c r}^{-}$) produces a "projector" onto the holomorphic (respectively antiholomorphic) discrete series.

Our next goal is to obtain a projector onto the continuous series component $\mathrm{P}^{\text {cont }}$. We expect that $\mathrm{P}^{\text {cont }}$ will be given by the same formula (10) but with a different choice of the cycle of integration, quite possibly supported in $\Omega \backslash\left(\Gamma^{0} \cup \overline{\Gamma^{0}}\right)$. To get $\mathrm{P}^{\text {cont }}$ we use a conformal map $\gamma: \mathbb{H}_{\mathbb{C}} \rightarrow \mathbb{H}_{\mathbb{C}}$ which sends

$$
\mathbb{H}_{\mathbb{R}} \tilde{\rightarrow} \mathbb{M} \quad \text { and } \quad S U(1,1) \stackrel{\sim}{\rightarrow} \widetilde{H}^{3} \quad \text { (with singularities) }
$$

where $\widetilde{H}^{3}$ is the unit hyperboloid of one sheet in $\mathbb{M}$; we call $\gamma$ the "Cayley transform". The hyperboloid $\widetilde{H}^{3}$ can be identified with $S L(2, \mathbb{C}) / S U(1,1)$ and is usually called the imaginary Lobachevski space. The group $S L(2, \mathbb{C})$ acts naturally on $L^{2}\left(\widetilde{H}^{3}\right)$ and decomposes into the discrete and continuous components:

$$
L^{2}\left(\widetilde{H}^{3}\right) \simeq L_{\text {discr }}^{2}\left(\widetilde{H}^{3}\right) \oplus L_{\text {cont }}^{2}\left(\widetilde{H}^{3}\right)
$$

as representations of $S L(2, \mathbb{C})$ (see, for example, [GGV]). Then the map $\gamma$ switches the discrete and continuous components:

$$
L_{\text {cont }}^{2}(S U(1,1)) \simeq L_{\text {discr }}^{2}\left(\widetilde{H}^{3}\right), \quad L_{\text {discr }}^{2}(S U(1,1)) \simeq L_{\text {cont }}^{2}\left(\widetilde{H}^{3}\right) .
$$

This explains the purpose of the Cayley transform - it is easier to find the projector onto the discrete component than onto the continuous one! Once the projector onto $L_{d i s c r}^{2}\left(\widetilde{H}^{3}\right)$ is found we can pull it back to $\mathbb{H}_{\mathbb{R}}$ to get $\mathrm{P}^{\text {cont }}$. We expect it to have the form

$$
\left(\mathrm{P}^{\text {cont }}\right) f\left(X_{0}\right)=\frac{1}{2 \pi^{2}} \int_{C_{\text {cont }}} \frac{\left(Z-X_{0}\right)^{-1}}{N\left(Z-X_{0}\right)} \cdot D z \cdot f(Z),
$$

where $C_{\text {cont }}$ is a certain three cycle in $\Omega$.

The integral formulas for $\mathrm{P}^{\text {discr }}$ and $\mathrm{P}^{\text {cont }}$ strongly suggest that the separation of the series is a topological phenomenon! Thus there is some underlying homology theory still waiting to be developed. We hope that this geometric analytic realization of the separation of the series will extend to higher rank groups. Such a picture fits well into the Gelfand-Gindikin program initiated in [GG which realizes representations of reductive groups $G$ in function spaces of open domains in $G_{\mathbb{C}}$ (complexification of $G$ ). This geometric relationship between quaternionic analysis and the separation of the series for $S L(2, \mathbb{R})$ will be the subject of our upcoming paper FL2]. 


\section{References}

[ACCK] N. Arkani-Hamed, F. Cachazo, C. Cheung, J. Kaplan, The S-Matrix in Twistor Space, arXiv:0903.2110 (2009).

[BDS] F. Brackx, R. Delanghe, F. Sommen, Clifford Analysis, Pitman, London, 1982.

[Co] J. Cockle, On Systems of Algebra Involving More than One Imaginary, Philosophical Magazine (series 3) 35 (1849), 434-435.

[CSSS] F. Colombo, I. Sabadini, F. Sommen, D. C. Struppa, Analysis of Dirac systems and computational algebra, Progress in Mathematical Physics, vol. 39, Birkhäuser Boston, 2004 .

[DSS] R. Delanghe, F. Sommen, V. Souček, Clifford algebra and spinor-valued functions, Kluwer Academic Publishers Group, Dordrecht, 1992.

[FL1] I. Frenkel, M. Libine, Quaternionic analysis, representation theory and physics, Advances in Math 218 (2008), 1806-1877; also arXiv:0711.2699.

[FL2] I. Frenkel, M. Libine, Split quaternionic analysis and the separation of the series for $S L(2, \mathbb{R})$ and $S L(2, \mathbb{C}) / S L(2, \mathbb{R})$, work in progress.

[F1] R. Fueter, Die Funktionentheorie der Differentialgleichungen $\Delta u=0$ und $\Delta \Delta u=0$ mit vier reellen Variablen, Comment. Math. Helv. 7 (1934), no. 1, 307-330.

[F2] R. Fueter, Über die analytische Darstellung der regulären Funktionen einer Quaternionenvariablen, Comment. Math. Helv. 8 (1935), no. 1, 371-378.

[GG] I. M. Gelfand, S. G. Gindikin, Complex manifolds whose spanning trees are real semisimple Lie groups, and analytic discrete series of representations, Funktsional. Anal. i Prilozhen. 11 (1977), no. 4, 19-27, 96.

[GGV] I. M. Gelfand, M. I. Graev, N. Ya. Vilenkin, Generalized Functions. Vol. 5: Integral Geometry and Representation Theory, translated from the Russian by E. Saletan, Academic Press, New York and London 1966.

[GM] J. Gilbert, M. Murray, Clifford Algebras and Dirac Operators in Harmonic Analysis, Cambridge Univ. Press, Cambridge, UK, 1991.

[GT] F. Gürsey, C.-H. Tze, On the role of division, Jordan and related algebras in particle physics, World Scientific Publishing Co., 1996.

[KoØ] T. Kobayashi, B. Ørsted, Analysis on the minimal representation of $O(p, q)$ I, II, III, Adv. Math. 180 (2003), no. 2, 486-512, 513-550, 551-595.

$[\mathrm{Ku} \varnothing] \quad \mathrm{K}$. Koufany, B. Ørsted, Function spaces on the Ol'shanskiน semigroup and the Gel'fand-Gindikin program, Ann. Inst. Fourier (Grenoble) 46 (1996), no. 3, 689-722.

[MS] L. Mason, D. Skinner, Scattering Amplitudes and BCFW Recursion in Twistor Space, arXiv:0903.2083 (2009).

[R] J. Ryan, Complexified Clifford Analysis, Complex Variables Theory Appl. 1 (1982/83), no. 1, 119-149. 
[St] R. S. Strichartz, Harmonic analysis on hyperboloids, J. Functional Analysis 12 (1973), 341-383.

[Su] A. Sudbery, Quaternionic analysis, Math. Proc. Camb. Math. Soc. 85 (1979), 199225.

E-mail address: mlibine@indiana.edu

Department of Mathematics, Indiana University, Rawles Hall, 831 East 3rd St, Bloomington, IN 47405 